\title{
Euler-Maclaurin Closed Form Finite State Space Model for a String Applied to Broadband Plate Vibrations
}

\author{
Michael J. Panza \\ Mechanical Engineering, Gannon University, 109 University Square, Erie, PA 16541, USA \\ Correspondence should be addressed to Michael J. Panza, panza@gannon.edu
}

Received 29 July 2009; Revised 9 February 2010; Accepted 2 March 2010

Academic Editor: Paulo Batista Gonçalves

Copyright (C) 2010 Michael J. Panza. This is an open access article distributed under the Creative Commons Attribution License, which permits unrestricted use, distribution, and reproduction in any medium, provided the original work is properly cited.

The Euler-Maclaurin sun formula is applied to the infinite series Green's function solution in the space-time Laplace transform domain for the one dimensional wave equation for a string fixed at each end. The resulting approximate closed form solution is used to derive a single third order input-output ordinary differential equation to model the string dynamics. The average modal density of a plate is shown to be comparable to a string. A finite three state-space model is developed for the string and applied to the vibrations of a plate subjected to broadband random and impulse inputs. The applications include the direct problem of determining the response to a disturbance input and the inverse problem of identifying the disturbance input with a finite state observer based on the finite string model. Numerical simulations using many plate modes are obtained in the time and frequency domains and are used to compare the multimodal plate model to the finite string based model and to demonstrate how the finite string based model can be used to represent the multimodal vibrations of the plate.

\section{Introduction}

The broadband high-frequency analysis of distributed parameter systems governed by partial differential equations usually requires a very large multimodal model, a many-node discrete grid finite difference or finite element model, or a statistical energy analysis (SEA) approach. For multimodal models, most work to date has been for disturbance inputs such as impulse type or a low-frequency random type. For example, disturbance force identification problems for structures such as beams and plates using a multimodal model of vibration have been the subject of studies $[1,2]$ where several lower-order modes are used to represent the structure subjected to either an impulse-type input or a low-frequency random-type input. These reduced order models are usually sufficient for frequencies less than about $200 \mathrm{~Hz}$. A novel discrete high-frequency forced vibration method using a large number of plate modes and based on the high-frequency plate modal density was applied for a harmonic force varied 
over a wide range of frequencies [3]. A large order discrete grid space time model for the free vibrations of string and cable structures is well suited for computer solutions [4] but there must be a very large number of spatial nodes to provide accurate vibration responses for high frequencies. Finite element models provide direct computer solutions especially for shocktype inputs where the lower modes dominate the response [5]. SEA methods are suitable for higher frequencies where many modes participate in the response and are usually applied to high-frequency random vibration which results in audible noise radiation typically above $200 \mathrm{~Hz}[6,7]$. The statistical nature of the method adds a degree of uncertainty to the results.

Although the three model methods discussed above are quite good for a range of applications where vibration and noise response are the primary goal of the model, they must be selected and formed to suit the specific application and frequency range and are somewhat complicated for use in the design of control systems or observers. A small-order finite state space model that can encompass a broad frequency range from very low to very high may be a useful alternative when design considerations are important. There is a dearth of work to develop small-order finite state space models for distributed parameter systems where design considerations for a broad range of frequencies are required in both vibration and noise problems. A simple model that is applicable for both low-frequency vibration due to shocktype inputs and high-frequency vibration due to broadband random type inputs can be a good alternative to the three more sophisticated models (multimodal, discrete/ finite element, and SEA) discussed above. Recent studies [8-10] have shown that the Euler-Maclaurin sum formula can be used to provide an approximate closed form representation for the infinite space-time series that results from the solution to distributed parameter systems governed by the wave equation.

This paper has two main objectives. First is to show how the approximate closed form solution to the wave equation for a string fixed on each end obtained from the EulerMaclaurin sum formula can be transformed into a low-order finite state space form. Second is to show how this finite state space model can be used in the dynamics of a plate, a task motivated by the approximate similarity of the mode spacing for a string and a plate. The Euler-Maclaurin sum formula is applied to obtain an approximate closed form Green's function for the infinite series solution of a string. A third-order finite state space model is developed from the approximate solution and compared to a multimodal model of a simply supported plate for a broadband random input and an impulse input. The string model is then used in a finite Leuenberger observer for estimating a broadband disturbance applied to a plate. The justification for using a string model for problems of multimodal broadband frequency vibration of a plate stems from a comparison of the natural frequency spacing of a string and a plate with the same fundamental frequency. The average modal density $n(f)$ at high enough frequencies $(f)$ of a thin plate of any shape, thickness $h$, surface area $S$, and longitudinal wave speed $c_{L}$ is compared to the fundamental frequency $f_{1}$ of a square simply supported plate with a $v=0.3$ Poisson's ratio [11],

$$
n(f)=\frac{\sqrt{12} S}{2 h c_{L}}, \quad f_{1}=\frac{\pi h c_{L}}{\sqrt{12} S \sqrt{1-v^{2}}} \longrightarrow \Delta f=\frac{1}{n(f)}=0.61 f_{1}
$$

Thus the average modal frequency spacing $\Delta f$ of a plate compares to some degree to the frequency spacing $\Delta f_{\text {string }}=f_{1}$ for a string. The Euler-Maclaurin finite string model may provide a reasonable alternative to a multimodal plate model with the same fundamental frequency. 


\section{Approximate Green's Function for String Wave Equation}

We consider a string with fixed ends, length $L$, mass $M$, and wave speed $c$. The Green function $g(x, t)$ satisfies the following partial differential equation and boundary conditions with $\delta$ being the Dirac delta function

$$
c^{2} \frac{\partial^{2} g}{\partial x^{2}}-\frac{\partial^{2} g}{\partial t^{2}}=-\frac{L}{M} \delta\left(x-x_{o}\right) \delta(t), \quad g=0 @ x=0, L
$$

Defining $G(x, s)$ as the $t$ domain Laplace transform of $g(x, t)$, the solution to (2.1) in the complex $s$ domain can be expressed as an infinite series of eigenfunctions (modes) with natural frequencies $\omega_{n}=n \omega_{1}$

$$
G(x, s)=\frac{2}{M} \sum_{n=1}^{\infty} \sin \left(\frac{n \pi x}{L}\right) \sin \left(\frac{n \pi x_{o}}{L}\right)\left(\frac{1}{s^{2}+\omega_{n}^{2}}\right) .
$$

With the fundamental mode $n=1$ separated out, (2.2) can be expressed in the form

$$
\begin{aligned}
G(x, s)= & \frac{2}{M} \sin \left(\frac{\pi x}{L}\right) \sin \left(\frac{\pi x_{o}}{L}\right)\left(\frac{1}{s^{2}+\omega_{1}^{2}}\right) \\
& -\frac{1}{M} \sum_{n=2}^{\infty} \cos \left[\frac{n \pi\left(x+x_{o}\right)}{L}\right]\left(\frac{1}{s^{2}+\omega_{n}^{2}}\right)+\frac{1}{M} \sum_{n=2}^{\infty} \cos \left[\frac{n \pi\left(x-x_{o}\right)}{L}\right]\left(\frac{1}{s^{2}+\omega_{n}^{2}}\right) .
\end{aligned}
$$

Defining $a_{1}=\pi\left(x+x_{0}\right) / L$ and $a_{2}=\pi\left(x-x_{0}\right) / L$, the sums in (2.3) have the form

$$
S_{i}=\sum_{n=2}^{\infty} \frac{\cos \left(n a_{i}\right)}{s^{2}+n^{2} \omega_{1}^{2}}
$$

The following form of the Euler-Maclaurin sum formula from Apostol [12] is applied to the sum in (2.4)

$$
\sum_{n=2}^{\infty} f_{n}=\int_{2}^{\infty} f(\mu) d \mu+\left(\frac{1}{2}\right)[f(2)+f(\infty)]+\int_{2}^{\infty} \frac{\partial f}{\partial \mu}\left(\mu-[\mu]-\frac{1}{2}\right) d \mu,
$$

where the brackets [ ] represent the smallest integer part. Since the goal of this paper is obtaining an approximate closed form solution, the second integral is considered as a remainder $R(x, s)$ with an upper bound given by

$$
\begin{aligned}
R_{\operatorname{Max}_{i}} & =\left|\int_{2}^{\infty} \frac{\partial f}{\partial \mu}\left(\mu-[\mu]-\frac{1}{2}\right) d \mu\right| \leq\left[\operatorname{Max}\left|\mu-[\mu]-\frac{1}{2}\right|\right]\left|\int_{2}^{\infty} \frac{\partial f}{\partial \mu} d \mu\right| \\
& =\frac{1}{2}\left|\int_{2}^{\infty} d f\right|=\frac{1}{2}|f(\infty)-f(2)|=\frac{\cos \left(2 a_{i}\right)}{2\left(s^{2}+4 \omega^{2}\right)} .
\end{aligned}
$$


The main part of the Euler-Maclaurin sum in (2.5) is the integral which is split up into two parts

$$
\int_{2}^{\infty} f(\mu) d \mu=\int_{0}^{\infty} \frac{\cos \left(\mu a_{i}\right)}{s^{2}+\mu^{2} \omega_{1}^{2}} d \mu-\int_{0}^{2} \frac{\cos \left(\mu a_{i}\right)}{s^{2}+\mu^{2} \omega_{1}^{2}} d \mu
$$

The first integral in (2.7) is considered dominant and has a closed form solution from the lists of Fourier transforms given by Oberhettinger [13]

$$
\int_{0}^{\infty} \frac{\cos \left(\mu a_{i}\right)}{s^{2}+\mu^{2} \omega_{1}^{2}} d \mu=\frac{\pi}{2 \omega_{1} s}\left[\mathrm{e}^{-\left(s a_{i} / \omega_{1}\right)} U\left(a_{i}\right)+\mathrm{e}^{\left(s a_{i} / \omega_{1}\right)} U\left(-a_{i}\right)\right]
$$

where $U$ is the unit-Heaviside step function.

The second integral in (2.7) is considered minor compared to the first. Combining all the terms in (2.5)-(2.8), the Euler-Maclaurin approximate closed form solution for Green's function is given by

$$
\begin{aligned}
M G(x, s)= & 2 \sin \left(\frac{\pi x}{L}\right) \sin \left(\frac{\pi x_{o}}{L}\right)\left(\frac{1}{s^{2}+\omega_{1}^{2}}\right) \\
& +\sum_{i=1}^{2}(-1)^{i}\left\{\frac{\pi}{2 \omega_{1} s}\left[\mathrm{e}^{-\left(s a_{i} / \omega_{1}\right)} U\left(a_{i}\right)+\mathrm{e}^{\left(s a_{i} / \omega_{1}\right)} U\left(-a_{i}\right)\right]+\frac{\cos \left(2 a_{i}\right)}{2\left(s^{2}+4 \omega_{1}^{2}\right)}\right\} \\
& +\int_{0}^{2} \frac{\cos \left(\mu a_{1}\right)-\cos \left(\mu a_{2}\right)}{s^{2}+\mu^{2} \omega_{1}^{2}} d \mu+R_{\max _{2}}-R_{\max _{1} .}
\end{aligned}
$$

For the work in this paper, $G$ is simplified by considering the point $x=x_{0}=L / 2$ which gives $a_{1}=\pi$ and $a_{2}=0$ and cancels the $R_{\max }$ terms and the two $\cos \left(2 a_{i}\right)$ terms. The finite integral is considered small and may be approximately evaluated as

$$
\begin{aligned}
-\int_{0}^{2} \frac{d \mu}{s^{2}+\mu^{2} \omega_{1}^{2}}+\int_{0}^{2} \frac{\cos (\mu \pi) d \mu}{s^{2}+\mu^{2} \omega_{1}^{2}} & \cong-\int_{0}^{2} \frac{d \mu}{s^{2}+\mu^{2} \omega_{1}^{2}}+\int_{0}^{2} \frac{[-1 \text { to } 1] d \mu}{s^{2}+\mu^{2} \omega_{1}^{2}} \\
& \cong-\int_{0}^{2} \frac{d \mu}{s^{2}+\mu^{2} \omega_{1}^{2}}+\left[-\int_{0}^{2} \frac{d \mu}{s^{2}+\mu^{2} \omega_{1}^{2}} \text { to } \int_{0}^{2} \frac{d \mu}{s^{2}+\mu^{2} \omega_{1}^{2}}\right] \\
& \cong-2 \int_{0}^{2} \frac{d \mu}{s^{2}+\mu^{2} \omega_{1}^{2}} \text { to } 0 .
\end{aligned}
$$

Green's function in (2.9) becomes

$$
M G(s)=\left(\frac{2}{s^{2}+\omega_{1}^{2}}\right)+\frac{\pi}{2 \omega_{1} s}\left(1-\mathrm{e}^{-\left(\pi s / \omega_{1}\right)}\right)+\left[-\frac{2}{\omega_{1} s} \tan ^{-1}\left(\frac{2 \omega_{1}}{s}\right) \text { to } 0\right]
$$




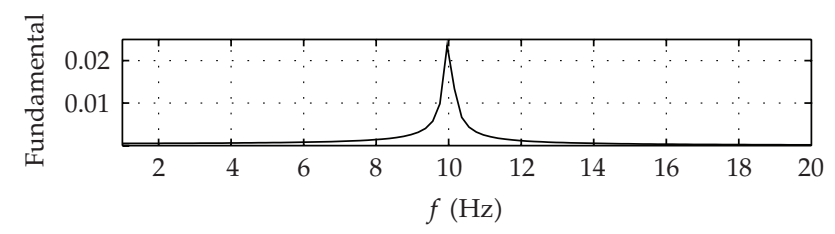

(a)

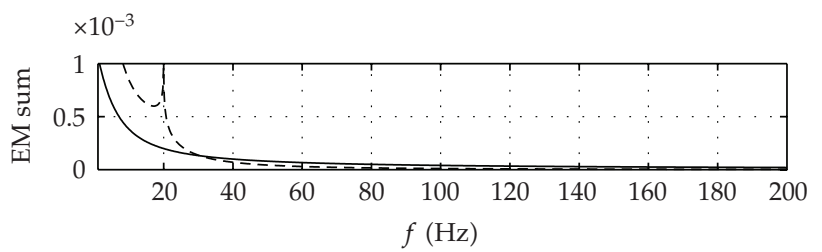

(b)

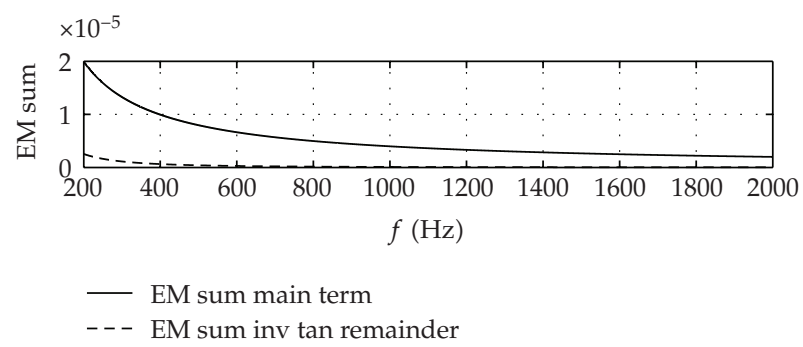

(c)

Figure 1: Frequency response for EM result for three ranges: (a) Fundamental for 0-20 Hz, (b) EM sum for 0-200 Hz, and (c) EM sum for 200-2000 Hz.

\section{Finite State Space Model from Approximate Green's Function}

The Euler-Maclaurin approximate form of Green's function from (2.11) is used for determining a closed form finite state space model for the string. Since the state space model is most useful in the time domain, numerical simulations will be presented in the time domain. Since the focus of the simulations for the random input is the steady state response, a damping term is included for the fundamental mode to allow the transient response dominated by this mode to be suppressed after a reasonably short time. Equation (2.11) becomes

$$
M G(s)=\left(\frac{2}{s^{2}+2 s_{1} \omega_{1} s+\omega_{1}^{2}}\right)+\frac{\pi}{2 \omega_{1} s}\left(1-\mathrm{e}^{-\left(\pi s / \omega_{1}\right)}\right)+\left[-\frac{2}{\omega_{1} s} \tan ^{-1}\left(\frac{2 \omega_{1}}{s}\right) \text { to } 0\right] .
$$

As presented, it is a fifth-order system in $s$. To obtain a more mathematically tractable function that contains the major components and can be transformed into one input/output ordinary differential equation, $M G(s)$ is further simplified by neglecting the inverse tangent term. This simplification is reasonable, especially for the high frequencies of interest, since the inverse tangent term is considered minor as it comes from the integral from 0 to 2 in the Euler-Maclaurin integral of (2.7). Figure 1 shows a comparison of the magnitude of the frequency response $(s=j \omega)$ of the inverse tangent term and the first two terms of (3.1). 
For higher frequencies, $f \gg f_{1}$, the inverse tangent term is small enough to be neglected. The system in (3.1) is reduced to a third-order system in s. Defining an impulse response function $H(s)=M G(s),(3.1)$ can be written as

$$
\begin{aligned}
& 2 \omega_{1} s^{3} H(s)+4 \zeta_{1} \omega_{1}^{2} s^{2} H(s)+2 \omega_{1}^{3} s H(s) \\
& \quad=4 \omega_{1} s+\pi\left(s^{2}+\omega_{1}^{2}\right)-\pi\left(s^{2}+\omega_{1}^{2}\right) \mathrm{e}^{-\left(\pi s / \omega_{1}\right)}+2 \zeta_{1} \omega_{1} s \pi\left(1-e^{-\left(\pi s / \omega_{1}\right)}\right) .
\end{aligned}
$$

Applying the inverse Laplace $s \rightarrow t$ gives a third-order differential equation for the impulse response function $h(t)$. Using primes to denote time derivatives,

$$
\begin{aligned}
2 \omega_{1} h^{\prime \prime \prime}(t)+4 \zeta_{1} \omega_{1}^{2} h^{\prime \prime}(t)+2 \omega_{1}^{3} h^{\prime}(t)= & \pi \omega_{1}^{2} \delta(t)+4 \omega_{1} \delta^{\prime}(t)+\pi \delta^{\prime \prime}(t)-\pi \omega_{1}^{2} \delta\left(t-\frac{\pi}{\omega_{1}}\right) \\
& -\pi \delta^{\prime \prime}\left(t-\frac{\pi}{\omega_{1}}\right)+2 \zeta_{1} \omega_{1} \pi \delta^{\prime}(t)-2 \zeta_{1} \omega_{1} \pi \delta^{\prime}\left(t-\frac{\pi}{\omega_{1}}\right) .
\end{aligned}
$$

For the one input/output differential equation that may be used to model the string dynamics, consider output $y(t)$, input $u(t)$, and input with delay $\bar{u}(t)=u\left(t-\pi / \omega_{1}\right)$. Equation (3.3) becomes

$$
\begin{aligned}
2 \omega_{1} y^{\prime \prime \prime}(t)+4 \zeta_{1} \omega_{1}^{2} y^{\prime \prime}(t)+2 \omega_{1}^{3} y^{\prime}(t)= & \pi \omega_{1}^{2} u(t)+4 \omega_{1} u^{\prime}(t)+\pi u^{\prime \prime}(t)-\pi \omega_{1}^{2} \bar{u}(t)-\pi \bar{u}^{\prime \prime}(t) \\
& +2 \zeta_{1} \omega_{1} \pi u^{\prime}(t)-2 \zeta_{1} \omega_{1} \pi \bar{u}^{\prime}(t) .
\end{aligned}
$$

Using constants $\beta_{0}, \alpha_{0}, \beta_{1}$, and $\alpha_{1}$, the following three state variables are considered

$$
x_{1}=y, \quad x_{2}=y^{\prime}-\beta_{0} u-\alpha_{0} \bar{u}, \quad x_{3}=x_{2}^{\prime}-\beta_{1} u-\alpha_{1} \bar{u} .
$$

The states are substituted into (3.4) to replace all state derivatives greater than one and the coefficients of all of like $u$ type terms are collected and set equal to zero. The resulting parameters are determined as

$$
\beta_{0}=\frac{\pi}{2 \omega_{1}}, \quad \alpha_{0}=-\frac{\pi}{2 \omega_{1}}, \quad \beta_{1}=2, \quad \alpha_{1}=0
$$

Thus the states become

$$
\begin{aligned}
& x_{1}=y, \\
& x_{2}=y^{\prime}-\frac{\pi}{2 \omega_{1}} u+\frac{\pi}{2 \omega_{1}} \bar{u}, \\
& x_{3}=y^{\prime \prime}-\frac{\pi}{2 \omega_{1}} u^{\prime}+\frac{\pi}{2 \omega_{1}} \bar{u}^{\prime}-2 u .
\end{aligned}
$$


From (3.5) and (3.7), the state equations are given by

$$
\begin{aligned}
\frac{d x_{1}}{d t} & =x_{2}+\frac{\pi}{2 \omega_{1}} u-\frac{\pi}{2 \omega_{1}} \bar{u}, \\
\frac{d x_{2}}{d t} & =x_{3}+2 u, \\
\frac{d x_{3}}{d t} & =-\omega_{1}^{2} x_{2}-2 \zeta_{1} \omega_{1} x_{3}-4 \zeta_{1} \omega_{1} u .
\end{aligned}
$$

In matrix-vector form, the finite third-order state space representation of the infinite modal dimension string developed from the application of the Euler-Maclaurin sum formula is given by

$$
\begin{aligned}
\mathbf{x}_{m} & =\left[\begin{array}{l}
x_{1} \\
x_{2} \\
x_{3}
\end{array}\right], \quad \frac{d \mathbf{x}_{m}}{d t}=\mathbf{A}_{m} \mathbf{x}_{m}+\mathbf{B}_{m} \mathbf{u}_{m}, \\
\mathbf{A}_{m} & =\left[\begin{array}{ccc}
0 & 1 & 0 \\
0 & 0 & 1 \\
0 & -\omega_{1}^{2} & -2 \zeta_{1} \omega_{1}
\end{array}\right], \quad \mathbf{B}_{m}=\left[\begin{array}{cc}
\frac{\pi}{2 \omega_{1}} & -\frac{\pi}{2 \omega_{1}} \\
2 & 0 \\
-4 \zeta_{1} \omega_{1} & 0
\end{array}\right], \quad \mathbf{u}_{m}=\left[\begin{array}{l}
u \\
\bar{u}
\end{array}\right] .
\end{aligned}
$$

\section{Comparison with Multimodal Plate Model}

The system plant for a simply supported rectangular plate $(a$ by $b$ ) is given by the infinite dimensional system with output $y$ and disturbance $f_{d}$ collocated at the center of the plate

$$
\begin{gathered}
\mathbf{x}_{p}=\left[\begin{array}{c}
\mathbf{q}_{n N \times 1} \\
\dot{\mathbf{q}}_{n N \times 1}
\end{array}\right], \quad \frac{d \mathbf{x}_{p}}{d t}=\mathbf{A}_{p} \mathbf{x}_{p}+\mathbf{B}_{p} f_{d}, \quad y=\mathbf{C}_{p} \mathbf{x}_{p}, \quad \mathbf{A}_{p_{2 N \times 2 N}}=\left[\begin{array}{cc}
\mathbf{0}_{N \times N} & \mathbf{I}_{N \times N} \\
-\boldsymbol{\omega}_{n N \times N}^{2} & -2 S_{n} \omega_{n N \times N}
\end{array}\right], \\
\omega_{n} \longrightarrow \omega_{i j}=\omega_{11}\left[\frac{(i / a)^{2}+(j / b)^{2}}{(1 / a)^{2}+(1 / b)^{2}}\right], \quad \mathbf{B}_{p_{2 N \times 1}}=\left[\begin{array}{c}
\mathbf{0}_{N \times 1} \\
-2 \sin \left(\frac{i \pi}{2}\right) \sin \left(\frac{j \pi}{2}\right)_{N \times 1}
\end{array}\right], \\
\mathbf{C}_{p}=\left[\sin \left(\frac{i \pi}{2}\right) \sin \left(\frac{j \pi}{2}\right)_{1 \times N}\right. \\
\left.\mathbf{0}_{1 \times N}\right],
\end{gathered}
$$

where $\mathbf{q}_{n}$ and $\dot{\mathbf{q}}_{n}$ are modal space vectors, $\mathbf{A}_{p}$ is the $2 N \times 2 N$ plant state matrix composed of four $N \times N$ submatrices, $\mathbf{B}_{p}$ is the $2 N \times 1$ plant input matrix for a simply supported plate composed of two $N \times 1$ vectors, $\mathrm{C}_{p}$ is the $1 \times 2 N$ output matrix, and $f_{d}$ is the disturbance input scalar. The plate natural frequencies are $\omega_{n}$ (with fundamental $\omega_{1}=\omega_{11}$ in (4.1)) and $\zeta_{n}$ is the modal damping factor considered equal for each mode greater than the fundamental. 


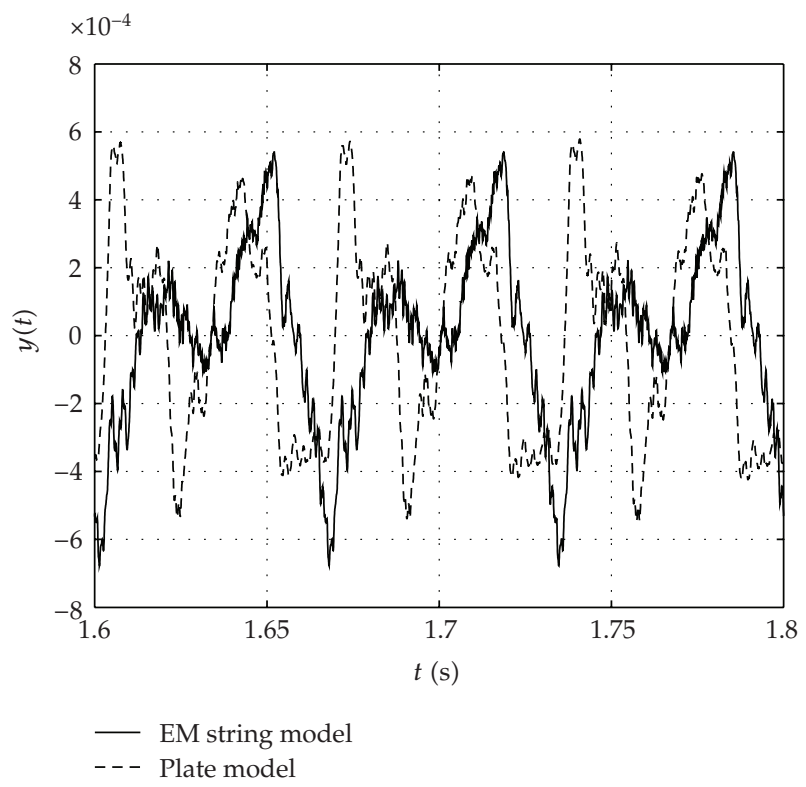

Figure 2: Comparison of steady state time response to random SPHS disturbance input of EM finite state space string model with the multimodal plate model.

In reality, $N \rightarrow \infty$ and the plant is an infinite dimensional system. Many modes will be used in simulations to show the utility of using the three-state model.

The disturbance input $f_{d}(t)$ used in the study is a broadband input generated with a multifrequency Schroeder-phased harmonic sequence (SPHS) [14] modified with random amplitudes to provide a simulated near broadband spectrum.

$$
f_{d}(t)=\sum_{k=1}^{N_{k}} A_{k} \sin \left(k \omega_{o} t+\phi_{k}\right), \quad \phi_{k}=\phi_{k-1}-2 \pi \sum_{j=1}^{k-1} \frac{A_{j}^{2}}{\sum_{k=1}^{N_{k}} A_{k}^{2}},
$$

where $\omega_{o}=2 \pi(15 \mathrm{~Hz}), N_{k}=200$ to provide an input from $15 \mathrm{~Hz}$ to $3000 \mathrm{~Hz}$, and $0 \leq A_{k} \leq 1$ via a uniformly distributed random number generator. This disturbance is random-like in time and periodic every 0.067 second due to the $15 \mathrm{~Hz}$ fundamental frequency. It provides a good model for a broadband multifrequency disturbance.

Figure 2 gives a comparison of the time response of the finite Euler-Maclaurin (EM) string model of (3.9) and the multimodal plate model of (4.1). The purpose of this figure is to compare the steady state behavior for the broadband random input. The plate model used for the comparison has an aspect ratio of 1.63, higher-order mode damping ratio of 0.01 , and approximately 200 modes up to $2000 \mathrm{~Hz}$. Both models are for a fundamental frequency of $10 \mathrm{~Hz}$ and a fundamental damping ratio of 1 . The critically damped fundamental mode is used to suppress its transient effect in a short period of time and allow the response to be mostly steady state in a reasonable amount of time. Without this large damping, the fundamental mode's transient response would still be dominant at the time used to show response at the time presented in the figure. Both the peak magnitude and the average magnitude compare quite well with rms values within $15 \%$. The average time between peaks and valleys is also close and both models show some high-frequency oscillations. The phase 


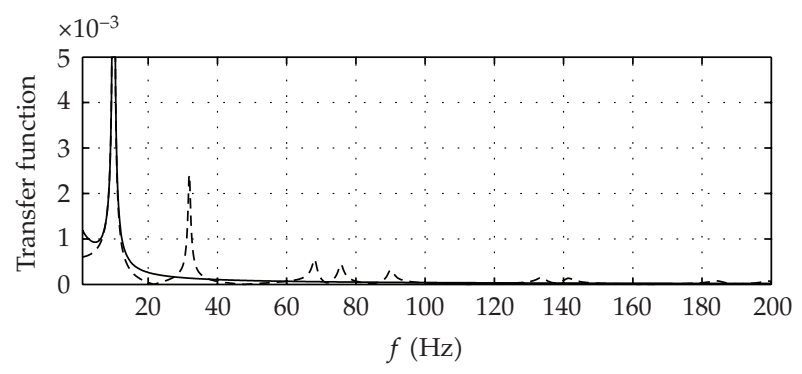

(a)

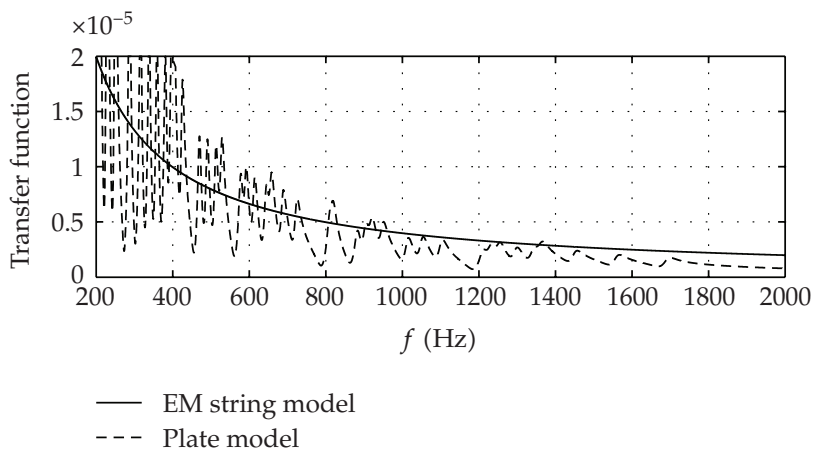

(b)

Figure 3: Comparison of the magnitude of the transfer function for the EM finite state space string model with the multimodal plate model, $\varsigma_{1}=0.01$ and $\varsigma_{n>1}=0.01$, for two frequency ranges: (a) $0-200 \mathrm{~Hz}$ and (b) 200-2000 Hz.

is a little different but this generally has no significance in the steady state. Overall, Figure 2 indicates that the EM string model is a reasonable approximation for a multimodal plate.

Separating out the fundamental string mode in (2.3) allows the EM string model to retain this important feature for other aspects of plate dynamics. Figure 3 gives a comparison of the magnitude of the transfer function for the EM finite state space string model of (3.9) with the multimodal plate model of (4.1). The damping factors are $\zeta_{1}=0.01$ and $\zeta_{n>1}=0.01$. Figure 4 gives a similar comparison for damping factors of $\zeta_{1}=0.01$ and $\zeta_{n>1}=0.05$. There is a very good match at the $10 \mathrm{~Hz}$ fundamental and for the drop off trend throughout the low- to high-frequency range. The primary difference between EM string and multimodal plate models is that the plate model provides the high-frequency oscillations relative to a common drop off trend for very small higher-order mode damping factors. This is expected because by design, the EM sum is applied to a lossless string system. The larger higher modal damping for Figure 4 shows how the two models are very close in the average drop off sense. Figure 5 gives a comparison of the transient response for the EM string and multimodal plate models to a 10 millisecond rectangular impulse shock. The damping factors are $\zeta_{1}=0.01$ and $\zeta_{n>1}=0.01$. Since the fundamental dominates such a response, the amplitudes and decay rates are comparable after the first peak. The only significant difference is that the EM string model gives a somewhat higher first peak while the plate model gives a slightly larger amplitude for the next three peaks and valleys. As indicated by the steady state response comparison shown in Figure 2 for the SPHS input, the basic transfer function comparison of Figures 3 and 4, and the similar transient response comparison of Figure 5, the EM finite string model derived for 


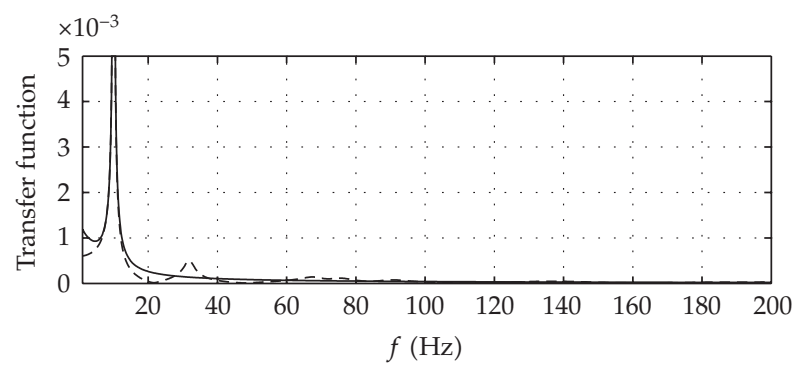

(a)

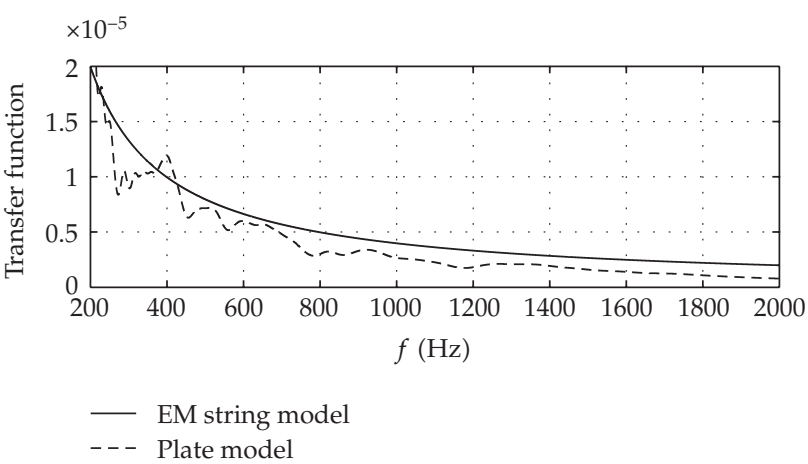

(b)

Figure 4: Comparison of the magnitude of the transfer function for the EM finite state space string model with the multimodal plate model, $\varsigma_{1}=0.01$ and $\varsigma_{n>1}=0.05$, for two frequency ranges: (a) $0-200 \mathrm{~Hz}$ and (b) 200-2000 Hz.

a lossless string with damping introduced for the fundamental system mode appears to be a reasonable finite state space model for a multimodal plate system.

\section{Inverse Dynamics Application for Estimating a Disturbance Input}

The states of the multimodal plate are too numerous to be estimated. As an alternative, the three EM model states are estimated with a Leunenberger observer [15] given by

$$
\frac{d \widehat{\mathbf{x}}_{m}}{d t}=\mathbf{A}_{m} \widehat{\mathbf{x}}_{m}+\mathbf{L}\left(y-\mathbf{C}_{m} \widehat{\mathbf{x}}_{m}\right),
$$

where $\widehat{\mathbf{x}}_{m}$ are the estimated states, $y=\mathbf{C}_{p} \mathbf{x}_{p}$ the output measurement of the actual plate, and $\mathbf{L}$ is a $3 \times 1$ observer matrix to be determined. The actual and model output vectors are given by

$$
\mathbf{C}_{p}=\left[\sin \left(\frac{\pi n}{2}\right)_{1 \times N} 0_{1 \times N}\right], \quad \mathbf{C}_{m}=\left[\begin{array}{lll}
1 & 0 & 0
\end{array}\right] .
$$

Combining (3.9)-(5.1) gives the state space for the combined plate, observer system

$$
\left[\begin{array}{c}
\frac{d \mathbf{x}_{p}}{d t} \\
\frac{d \widehat{\mathbf{x}}_{m}}{d t}
\end{array}\right]_{2 N+3 \times 1}=\left[\begin{array}{cc}
\mathbf{A}_{p 2 N \times 2 N} & \mathbf{0}_{2 N \times 3} \\
\mathbf{L C}_{p 3 \times 2 N} & \mathbf{A}_{m}-\mathbf{L C}_{m 3 \times 3}
\end{array}\right]_{2 N+3 \times 2 N+3}\left[\begin{array}{c}
\mathbf{x}_{p} \\
\widehat{\mathbf{x}}_{m}
\end{array}\right]+\left[\begin{array}{c}
\mathbf{B}_{p} \\
\mathbf{0}
\end{array}\right] f_{d} .
$$




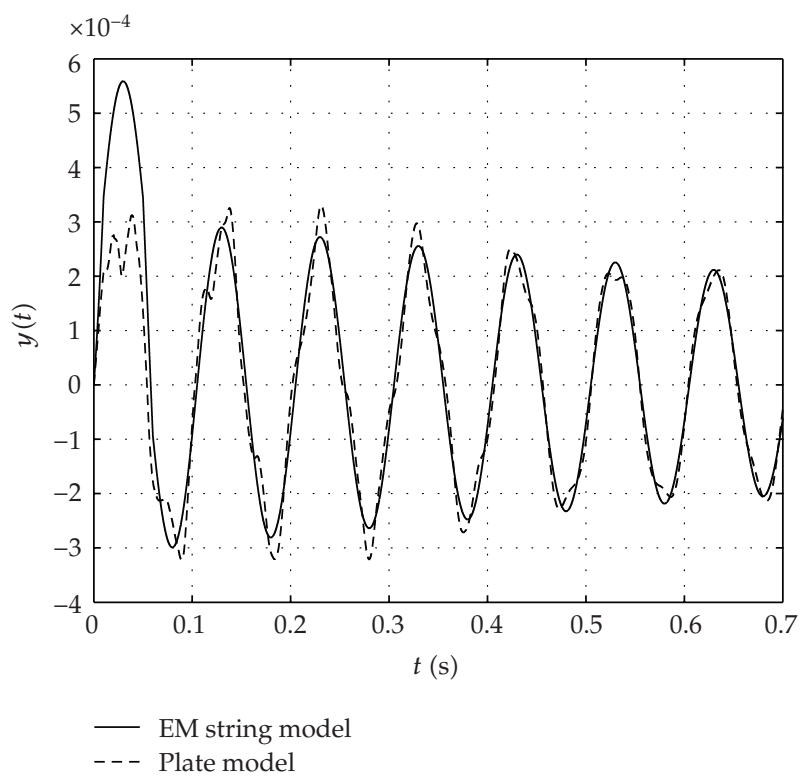

Figure 5: Comparison of the transient response for the EM finite state space string model with the multimodal plate model for a 10 millisecond square impulse shock.

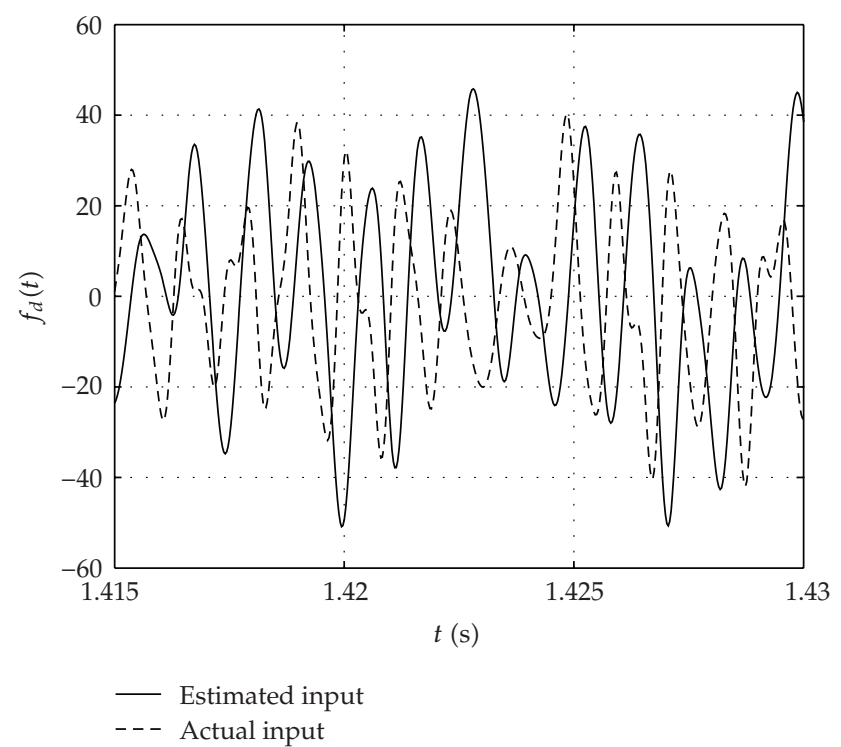

Figure 6: Comparison of the actual random SPHS disturbance input of (4.2) with the estimated input.

For a specific simulated disturbance input $f_{d},(5.3)$ is used to obtain the state estimate $\widehat{\mathbf{x}}_{m}$ for the finite third-order Euler-Maclaurin model. The finite Euler-Maclaurin model of (3.9) is used to determine the model based vector disturbance estimate $\widehat{\mathbf{u}}_{m}$. Solving (3.9) for $\widehat{\mathbf{u}}_{m}$, substituting $d \widehat{\mathbf{x}}_{m} / d t$ from (5.1), and simplifying give

$$
\widehat{\mathbf{u}}_{m}=\mathbf{B}_{m}^{-1} \mathbf{L}\left(\mathbf{C}_{p} \mathbf{x}_{p}-\mathbf{C}_{m} \widehat{\mathbf{x}}_{m}\right),
$$

where the generalized inverse of vector $\mathbf{B}_{m}$ is $\mathbf{B}_{m}^{-1}=\left(\mathbf{B}_{m}^{T} \mathbf{B}_{m}\right)^{-1} \mathbf{B}_{m}^{T}$. 
In (3.3), the presence of the delta function $\delta(t)$ and delay $\delta\left(t-\pi / \omega_{1}\right)$ implies that the disturbance estimate is the sum of the two elements of $\widehat{\mathbf{u}}_{m}$ and according to the definition in (3.9), it is given by

$$
\widehat{f}_{d}(t)=u(t)+\bar{u}(t)=u(t)+u\left(t-\frac{\pi}{\omega_{1}}\right)
$$

The estimated disturbance from (5.5) is compared to the actual disturbance applied to the system. This estimate is not directly measured but computed from an output measurement on the actual system and a finite dimension state space model obtained from the Euler-Maclaurin sum formula for the infinite series of terms in the system Green function. Simulations for the SPHS input will be used to show the accuracy of the procedure.

The Leuenberger matrix $\mathbf{L}=\left[\begin{array}{lll}L_{1} & L_{2} & L_{3}\end{array}\right]$ is determined by looking at the eigenvalues of the model observer matrix $\mathbf{A}_{m}-\mathbf{L C}_{m}$

$$
\left|\lambda \mathbf{I}-\left(\mathbf{A}_{m}-\mathbf{L C}_{m}\right)\right|=\left|\begin{array}{ccc}
\lambda+L_{1} & -1 & 0 \\
L_{2} & \lambda & -1 \\
L_{3} & \omega_{1}^{2} & \lambda
\end{array}\right|=\lambda^{3}+L_{1} \lambda^{2}+\left(L_{2}+\omega_{1}^{2}\right) \lambda+\left(L_{3}+\omega_{1}^{2} L_{1}\right)=0
$$

The desirable values of $\lambda$ are defined as $\lambda_{1}=-\alpha$ and $\lambda_{2,3}=-\alpha \pm i \omega_{1}$. The Leuenberger matrix components required to provide these desirable values are

$$
L_{1}=3 \alpha, \quad L_{2}=3 \alpha^{2}, \quad L_{3}=\alpha^{3}-2 \alpha \omega_{1}^{2}
$$

The basic procedure is to use plant model of (4.1) in the system equation (5.3) for design simulations to determine the best observer parameter $\alpha$ for a particular fundamental natural frequency $f_{1}$ and damping factors $\zeta_{n}$. In an actual situation for a plate with any boundary conditions or geometry, $f_{1}$ may be estimated with a finite element method and $\zeta_{n}$ may be estimated with a standard damping measurement. The disturbance estimate is then made by using the actual measured plant output $y$ in place of $\mathbf{C}_{p} \mathbf{x}_{p}$ in (5.4). The $\mathbf{C}_{m} \widehat{\mathbf{x}}_{m}$ term in (5.4) is still determined by integrating the system model equation (5.3) while using the plant model of (4.1). For the case of $f_{1}=10 \mathrm{~Hz}$ and $\zeta_{n>1}=0.05$, design simulations provide a best value of $\alpha=320$. Figure 6 gives a comparison of the actual SPHS disturbance input of (4.2) and the estimated disturbance from (5.5). A fundamental damping factor of $\zeta_{1}=1$ is used to provide a steady state condition at the time range given in the figure. Although phase is off, the distance between peaks and valleys and the amplitude of the oscillations, both of which are the important features in the steady state, are reasonably close for the actual and the estimated inputs. Figure 7 gives a comparison of the actual output $\mathbf{C}_{p} \mathbf{x}_{p}$ and the estimated output $\mathbf{C}_{m} \widehat{\mathbf{x}}_{m}$. The main difference is the existence of the small oscillations for the actual output.

\section{Conclusions}

A finite state space model has been shown to provide a reasonably accurate alternative to a multimodal model for a plate. The finite state space model retains the plate fundamental mode along with a finite part for the higher-order modes. This allows the model to be used 


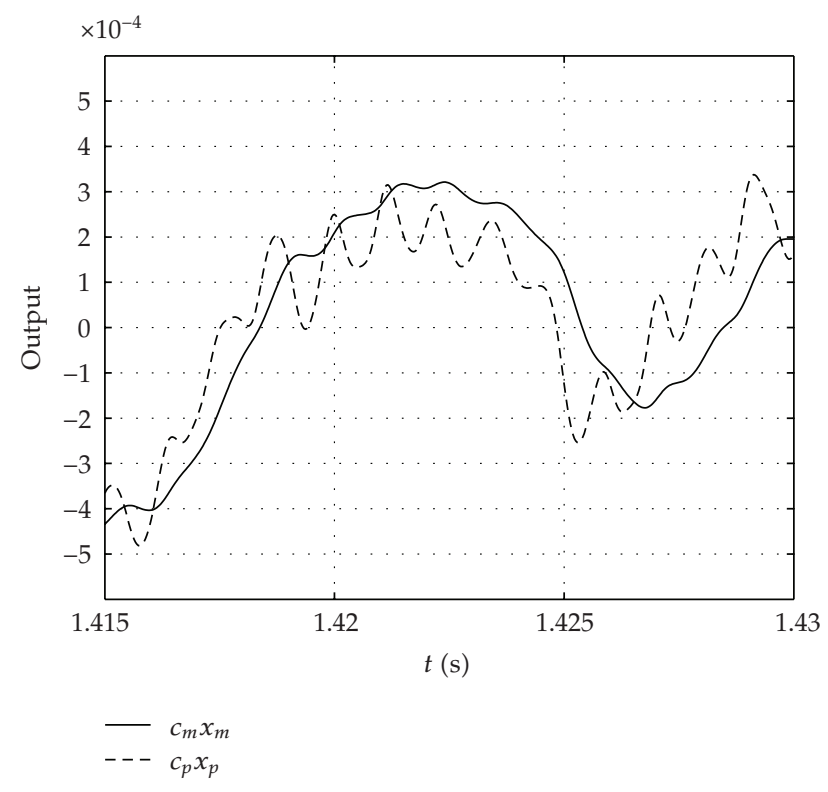

Figure 7: Comparison of the actual output $C_{p} x_{p}$ and the estimated output $C_{m} x_{m}$.

for a variety of situations. The paper uses the steady state response to a broadband randomlike input, the transient response to a shock-like input, and the steady state estimation of a broadband random-like disturbance to show the usefulness of the finite state space model. The finite state space model is derived from the dynamics of an elastic string fixed at each end. Starting with a partial differential equation description of the string dynamics, the infinite series form of the string Green function is transformed to an approximate closed form expression by applying the Euler-Maclaurin sum formula with remainder. A state space system consisting of three state variables is derived from the Euler-Maclaurin sum result. The finite state space description is applied directly to plate dynamic response analysis and is used in an inverse problem of estimating a disturbance input by applying a state observer based on the finite state space model. Comparative results indicate that the string-based finite state model can be a reasonable way to analyze the multimodal dynamics of a plate for several situations.

\section{References}

[1] R. Hashemi and M. H. Kargarnovin, "Vibration base identification of impact force using genetic algorithm," International Journal of Mechanical Systems Science and Engineering, vol. 1, no. 4, pp. 204-210, 2008.

[2] Z. R. Lu and S. S. Law, "Force identification based on sensitivity in time domain," Journal of Engineering Mechanics, vol. 132, no. 10, pp. 1050-1056, 2006.

[3] A. Seçgin and A. Saide Sarı̈ül, "A novel scheme for the discrete prediction of high-frequency vibration response: discrete singular convolution-mode superposition approach," Journal of Sound and Vibration, vol. 320, no. 4-5, pp. 1004-1022, 2009.

[4] A. Moshfegh, "Discrete state-space modeling for linear $n$ th-order constant coefficient distributedparameter systems," Dynamics and Control, vol. 3, no. 1, pp. 71-90, 1993.

[5] W.-H. Lee and S.-C. Han, "Free and forced vibration analysis of laminated composite plates and shells using a 9-node assumed strain shell element," Computational Mechanics, vol. 39, no. 1, pp. 41-58, 2006.

[6] D. Cui and I. A. Craighead, "Reduction in machine noise and vibration levels based on the statistical energy analysis method," Proceedings of the Institution of Mechanical Engineers, Part E, vol. 214, no. 3, pp. 147-155, 2000. 
[7] J. A. Steel, "A study of engine noise transmission using statistical energy analysis," Proceedings of the Institution of Mechanical Engineers, Part D, vol. 212, no. 3, pp. 205-213, 1998.

[8] M. J. Panza, "Closed form solution for acoustic wave equation between two parallel plates using Euler-Maclaurin sum formula," Journal of Sound and Vibration, vol. 277, no. 1-2, pp. 123-132, 2004.

[9] M. J. Panza, "Mathematical models for finite controllers for a class of bounded acoustic and structural dynamic systems," in Proceedings of the Active '04, pp. 20-22, Williamsburg, Va, USA, September 2004, paper no. a04_011.

[10] M. J. Panza, "Application of Euler-Maclaurin sum formula to obtain an approximate closed-form Green's function for a two-dimensional acoustical space," Journal of Sound and Vibration, vol. 311, no. 1-2, pp. 269-279, 2008.

[11] L. L. Beranek, Noise and Vibration Control, McGraw-Hill, New York, NY, USA, 1971.

[12] T. M. Apostol, "An elementary view of Euler's summation formula," The American Mathematical Monthly, vol. 106, no. 5, pp. 409-418, 1999.

[13] F. Oberhettinger, Fourier Transforms of Distributions and Their Inverses: A Collection of Tables, Probability and Mathematical Statistics, no. 1, Academic Press, New York, NY, USA, 1973.

[14] M. N. Sahinkaya, "Virtual non-linear disturbance observer by dual inverse dynamic modelling," Proceedings of the Institution of Mechanical Engineers, Part C, vol. 221, no. 6, pp. 677-688, 2007.

[15] B. Friedland, Control System Design, McGraw-Hill, New York, NY, USA, 1986. 


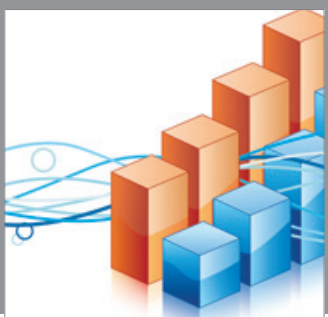

Advances in

Operations Research

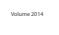

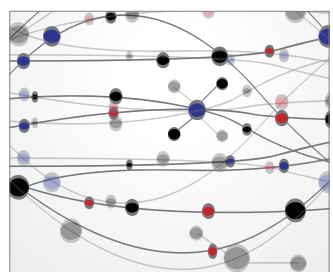

\section{The Scientific} World Journal
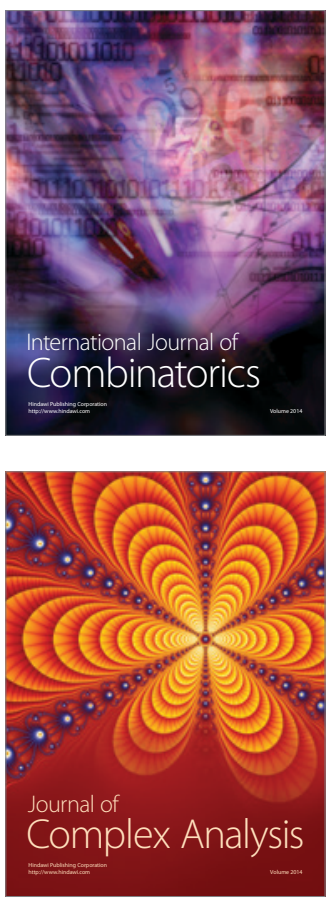

International Journal of

Mathematics and

Mathematical

Sciences
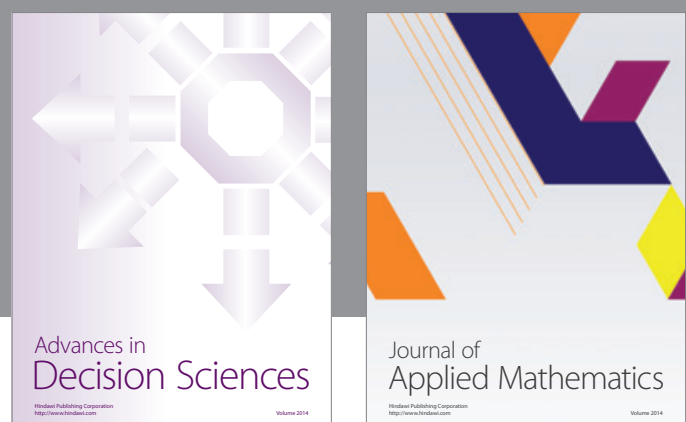

Journal of

Applied Mathematics
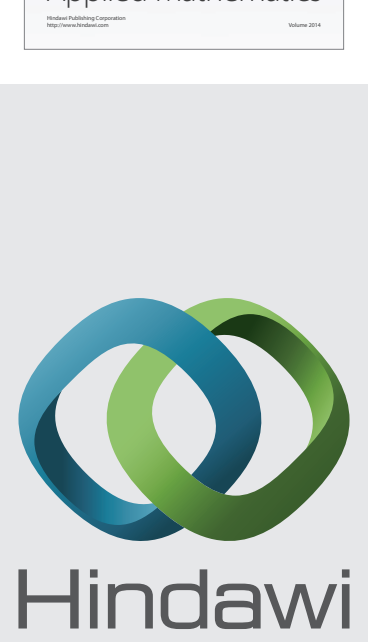

Submit your manuscripts at http://www.hindawi.com
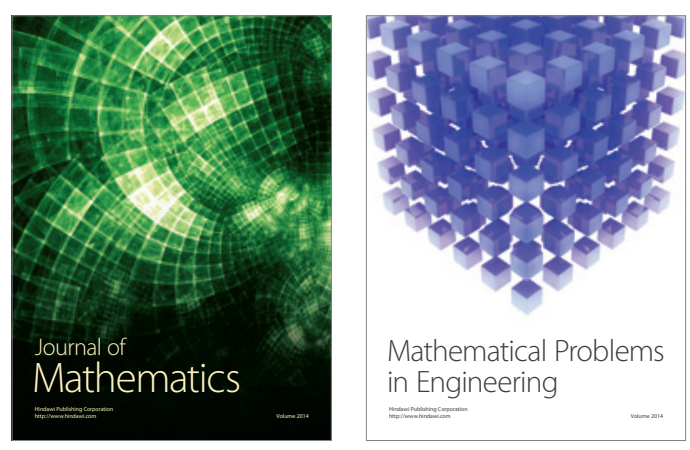

Mathematical Problems in Engineering
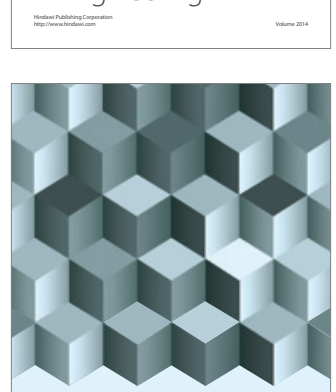

Journal of

Function Spaces
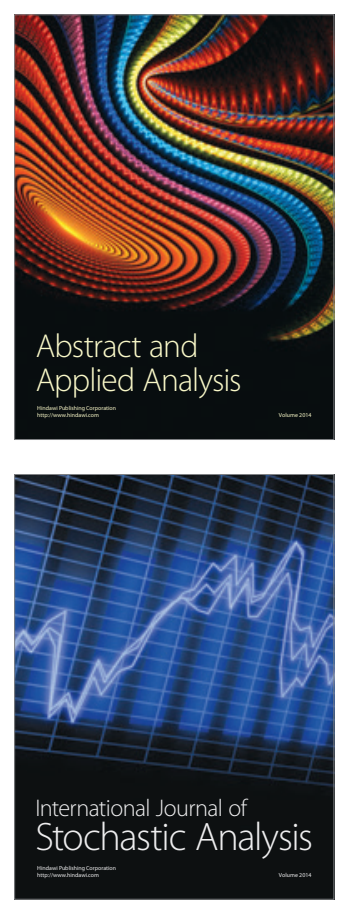

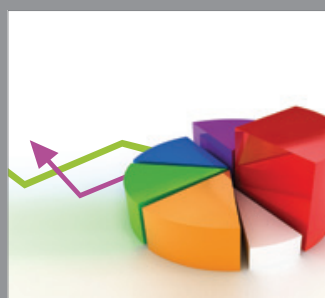

ournal of

Probability and Statistics

Promensencen
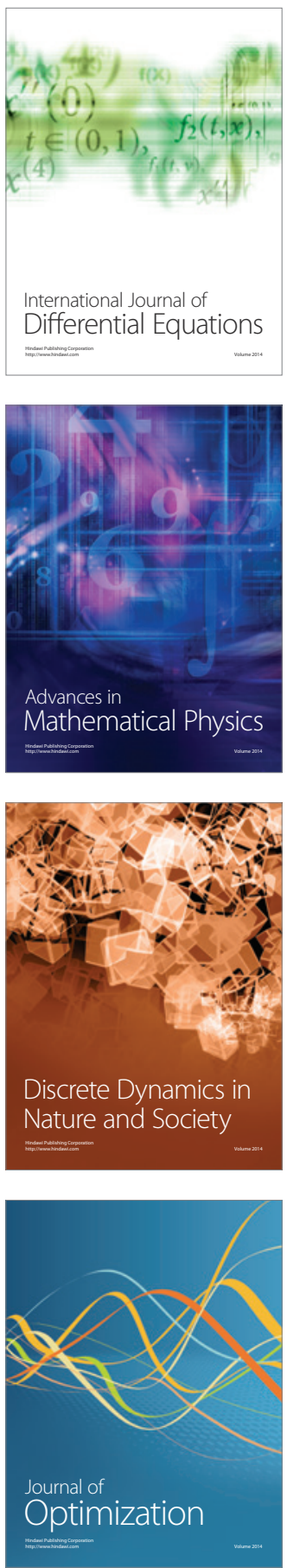